\title{
AN EXAMPLE OF NON-CONVEX MINIMIZATION AND AN APPLICATION TO NEWTON'S PROBLEM OF THE BODY OF LEAST RESISTANCE
}

\author{
T. LACHAND-ROBERT ${ }^{\mathrm{a}}$, M.A. PELETIER ${ }^{\mathrm{b}}$ \\ ${ }^{a}$ Université Pierre et Marie Curie, Laboratoire d'Analyse Numérique, 75252 Paris Cedex 05, France \\ ${ }^{\mathrm{b}}$ Centrum voor Wiskunde en Informatica P.O. Box 94079, 1090 GB Amsterdam, The Netherlands
}

Received in 20 January 2000, revised 10 May 2000

ABSTRACT. - We study the minima of the functional $\int_{\Omega} f(\nabla u)$. The function $f$ is not convex, the set $\Omega$ is a domain in $\mathbb{R}^{2}$ and the minimum is sought over all convex functions on $\Omega$ with values in a given bounded interval. We prove that a minimum $u$ is almost everywhere 'on the boundary of convexity', in the sense that there exists no open set on which $u$ is strictly convex. In particular, wherever the Gaussian curvature is finite, it is zero.

An important application of this result is the problem of the body of least resistance as formulated by Newton (where $f(p)=1 /\left(1+|p|^{2}\right)$ and $\Omega$ is a ball), implying that the minimizer is not radially symmetric. This generalizes a result in [1].

(C) 2001 L'Association Publications de l'Institut Henri Poincaré. Published by Elsevier B.V. All rights reserved

RÉSUMÉ. - On examine les minimums d'une fonctionnelle de la forme $\int_{\Omega} f(\nabla u)$ où $f$ n'est pas convexe et $\Omega$ est un domaine borné de $\mathbb{R}^{2}$, l'ensemble des fonctions admissibles $u$ étant restreint aux fonctions convexes à valeurs dans un intervalle fixé. On démontre que ces minimums sont presque partout à la limite de la convexité, en ce sens qu'il n'existe pas d'ouvert où ils sont strictement convexes. En particulier, aux points où leurs graphes possèdent une courbure gaussienne finie, celle-ci est nulle.

Ce résultat s'applique notamment au problème de la résistance minimale de Newton (où $f(p)=1 /\left(1+|p|^{2}\right)$ et $\Omega$ est une boule). Il implique que le minimum n'est pas à symétrie radiale, généralisant ainsi le résultat de [1].

(C) 2001 L'Association Publications de l'Institut Henri Poincaré. Published by Elsevier B.V. All rights reserved

\section{Introduction}

\subsection{Newton's problem}

The problem of the body of minimal resistance was introduced by Sir Isaac Newton in Principia Mathematica $[9,4]$ and his treatment has generally become known as one of

E-mail addresses: lachand@ann.jussieu.fr (T. Lachand-Robert), Mark.Peletier@cwi.nl (M.A. Peletier). 
the first examples of variational calculus. The problem can be stated mathematically in the following way: let $\Omega$ be a given domain (an open connected set) in $\mathbb{R}^{2}$, and minimize

$$
\int_{\Omega} \frac{d x}{1+|\nabla u(x)|^{2}}
$$

(where $|p|^{2}=p_{1}^{2}+p_{2}^{2}$ is the Euclidean norm) over the set $C_{M}$ of convex functions $u$ defined on $\Omega$ that are bounded above and below by constants 0 and $M>0$. Here the graph of $u$ is the shape of the body whose resistance is estimated by (1) under some simple physical assumptions. We refer the reader to [2] for a more detailed presentation of this problem, and some alternative ways to state it. It is also proved in [2] that the minimum is attained.

Newton computed a minimizing function $u_{\text {rad }}$ when $\Omega$ is a circular disc, assuming radial symmetry of the minimizer. Indeed, since the problem is invariant under rotation, one might reasonably expect that a minimizer has the same invariance. However, it was recently proved [1] that this function only minimizes (1) in the set of radially symmetric functions. This is related to the non-convex nature of (1), due to the fact that the Hessian matrix of the function $p \in \mathbb{R}^{2} \mapsto\left(1+|p|^{2}\right)^{-1}$ has a negative eigenvalue at every point; there is a 'non-radial' direction at $u_{\text {rad }}$ in which (1) has a negative second variation. Therefore we can achieve a lower value of the functional with nonradial functions. In particular it follows that the minimizers on a disc are not unique. Incidentally, P. Guasoni [5] has exhibited a non-radial function which achieves a lower value of the functional for $M \gtrsim 1.88$.

Unfortunately, the argument given in [1] provides no information on the shape of the minimizers other than the lack of radial symmetry. It has been conjectured by H. Berestycki that the minimizers could be 'affine by parts'. This conjecture arises from the following result:

LEMMA 1 [1, Remark 3.4]. - Assume that $u$ is of class $C^{2}$ in $\omega \subset \Omega$ and satisfies $\operatorname{det} d^{2} u>0$ in $\omega$. Then $u$ is not a minimizer of (1).

Proof. - The proof of this lemma is given in [1]; since it provides an insightful introduction to the results of this paper, we briefly describe the argument.

The crucial observation is that $u$ is 'interior' to the set $C_{M}$, in the sense that for any function $\phi \in C_{c}^{2}(\omega)$, the two functions $u \pm \varepsilon \phi$ both belong to $C_{M}$ if $\varepsilon>0$ is small enough. Therefore, if we assume that $u$ is a minimum, then $u$ satisfies the Euler equation

$$
\operatorname{div} d f(\nabla u)=0 \quad \text { in } \omega,
$$

where $f(p):=\left(1+|p|^{2}\right)^{-1}$, as well as

$$
\int_{\omega} d^{2} f(\nabla u) \nabla \phi \cdot \nabla \phi \geqslant 0,
$$

for all $\phi \in C_{c}^{2}(\omega)$. The idea is now to consider a function $\phi$ whose gradient is mainly oriented in the negative direction of the matrix $d^{2} f(\nabla u)$. Indeed, if $\omega$ is a sufficiently 
small set, we can assume that the matrices $\left\{d^{2} f(\nabla u)(x)\right\}_{x \in \omega}$ have a common negative direction. Supposing this direction to be along the first coordinate axis, we choose $\phi\left(x_{1}, x_{2}\right):=\eta(x) \sin \left(n x_{1}\right)$ where $\eta \in C_{c}^{2}(\omega)$ is a fixed function and $n$ is a sufficiently large number. It is now easy to verify that there is a contradiction with (3).

This lemma strongly suggests that in some sense a minimizer $u$ should satisfy $\operatorname{det} d^{2} u \equiv 0$. Unfortunately, the $C^{2}$-requirement is very strong, and nothing indicates that it is actually satisfied by a minimizer of Newton's problem. Even the radial solution is only smooth by parts (it has a flat circular zone where it is minimal, and the gradient is not continuous on its boundary). Moreover, 'most' convex functions are of class $C^{1}$ and strictly convex, but 'almost none' of them are $C^{2}$, in the sense of Baire category (see for instance $[13,14]$ and the references cited there). Since there is no regularizing effect in this sort of problems (see [7]), there is little hope that the minimizer is of class $C^{2}$.

Hence it is not clear what generalization of $\operatorname{det}^{2} u \equiv 0$ should hold for minimizers. We should note that the condition $\operatorname{det} d^{2} u \equiv 0$, even if it makes sense in some open subset $\omega \subset \Omega$, does not imply that $u$ is affine. It only indicates that the graph $u$ has vanishing Gauss curvature; hence $u$ could also be (locally) cylindrical or conical.

In this paper we investigate this question and prove that minimizers cannot be strictly convex in any open subset. (Throughout the paper, ' $u$ is strictly convex in $\omega$ ' means $\forall x, y \in \omega, \forall t \in(0,1), x \neq y \Rightarrow u(t x+(1-t) y)<t u(x)+(1-t) u(y)$.

As we remarked above, this behaviour is very unusual for a convex function, since 'most' convex functions are of class $C^{1}$ and strictly convex. Note that this result contains the statement of Lemma 1.

\subsection{The general problem}

We consider the problem

$$
\inf _{u \in \mathcal{C}} \int_{\Omega} f(\nabla u) d x,
$$

where $\Omega \subset \mathbb{R}^{2}$ is an open bounded domain, $f: \mathbb{R}^{2} \rightarrow \mathbb{R}_{+}$is a given nonnegative smooth function, and

$$
\mathcal{C}:=\{u: \Omega \rightarrow[0,1] ; u \text { convex }\} .
$$

This corresponds to the problem of the body of least resistance as stated above by changing $u$ to $u / M$ and setting $f(p)=1 /\left(M^{2}+|p|^{2}\right)$. Since the set $\mathcal{C}$ is compact in the $H_{\mathrm{loc}}^{1}(\Omega)$ topology [2] and $f$ is bounded from below, the minimum is always attained.

If $f$ is convex or concave, the problem has already been studied in [7] with a general constraint of the form (7). It is proved there that if $\underline{u}=\bar{u}$ on $\partial \Omega$, then the minimizer is equal to either $\underline{u}$ or $\bar{u}$, when these functions are convex. It was already explained in [7] that this sort of problem cannot be studied through the usual methods of the calculus of variations, due to the convexity constraint on $u$. In particular there is no 'regularizing effect' even if $f$ is convex.

In this paper we are interested in the case where $f$ is not convex but need not be concave either: the Hessian matrix $d^{2} f$ has at least one negative eigenvalue. If this is true for every $p$ in some subset $Q$ of $\mathbb{R}^{2}$ then we call $f$ nowhere convex in $Q$. As remarked before, the function $p \mapsto 1 /\left(1+|p|^{2}\right)$ considered by Newton is nowhere convex in $\mathbb{R}^{2}$. 
For technical reasons we have to sharpen this assumption and assume that $f$ satisfies Hypothesis $(\mathrm{H})$ on a set $Q \subset \mathbb{R}^{2}$ :

Hypothesis (H). - For all $V \in Q$ and all $W \in \mathbb{R}^{2}$,

$$
d^{2} f(V) W \cdot W=0=d^{3} f(V) W \cdot W \cdot W \Rightarrow W=0 .
$$

It is easy to verify that this condition is satisfied on $\mathbb{R}^{2}$ in the particular case $f(p)=1 /\left(M^{2}+|p|^{2}\right)$. Note that this hypothesis is used only in step 3 of the proof of Lemma 5 .

\subsection{Statement of main results}

Let us recall that a convex function $u$ is almost everywhere twice differentiable in its domain; moreover, it is actually of class $C^{1}$ on the set $\operatorname{dom}(\nabla u)$ where it is differentiable (see for instance [11], Theorem 25.5). If $A \subset \Omega$, we will note by $\nabla u(A)$ the image set of $A$ under the map $x \mapsto \nabla u(x)$, for those $x \in A$ where it is defined. We write conv $A$ for the convex hull of $A$.

Our main theorem is as follows:

THEOREM 1. - Let $u$ be a minimizer of Problem (4), and let $\Omega_{1}$ be an open convex subset of $\Omega$. If $f$ is nowhere convex and satisfies Hypothesis $(\mathrm{H})$ on conv $\nabla u\left(\Omega_{1}\right)$, then $u$ is not strictly convex on $\Omega_{1}$.

Note that this implies that the graph of $u$ contains at least one straight line segment in $\Omega_{1}$; by repeated application of this theorem it follows that there is a dense collection of line segments in the graph.

The proof of this statement will follow in Section 4. Let us first give some important consequences of this theorem.

First of all let us apply this result to the 'classical' problem as studied by Newton:

COROLlary 2. - Let $M>0$ and $f(p)=1 /\left(M^{2}+|p|^{2}\right)$, and let $\Omega_{1}$ be an open subset of $\Omega$. If $u$ solves Problem (4), then $u$ is not strictly convex in $\Omega_{1}$.

Observe that the radial minimizer calculated by Newton (which is strictly convex on a subset of the ball) cannot be a minimizer of (4), and consequently the minimizer of (4) is not radially symmetric. As mentioned before, this was already proved in [1] by a different argument.

Secondly, here is an alternative statement of Theorem 1:

COROLLARY 3. - Under the conditions of the theorem, if $\phi \in C^{0}(\Omega)$ is strictly convex in some open subset of $\Omega$, then $u-\phi$ is not convex.

(This follows from the theorem by observing that if $\phi$ is strictly convex in an open set $\Omega_{1}$, and $u-\phi$ is convex, then $u$ is strictly convex in $\Omega_{1}$.) Hence, $u$ is an 'almost extremal' point of $\mathcal{C}$, considered as a convex subset of the set of functions $C^{0}(\Omega)$.

It can be proved that if $u$ is a minimizer, then the sets

$$
N_{0}:=\{x \in \bar{\Omega} ; u(x)=0\}
$$


and $\{x \in \bar{\Omega} ; u(x)=1\}$ are non-empty; the latter is included in the boundary of $\Omega$, due to convexity. In the following, we do not examine the behaviour of $u$ near $N_{0}$, nor the shape of this set, since $u$ is not strictly convex in its interior; this question is studied in [8].

As a consequence, one can easily generalize the constraint ' $u(x) \in[0,1]$ ' to

$$
\underline{u}(x) \leqslant u(x) \leqslant \bar{u}(x),
$$

where $\underline{u}, \bar{u}$ are arbitrary functions (provided there exists at least one convex function satisfying (7)). In that case, $N_{0}$ is simply the set where equality is attained in one side of (7), and the theorem still holds if $\underline{u}, \bar{u}$ are affine functions, or if for instance $\Omega_{1} \cap N_{0}=\emptyset$.

More generally, the reader should note that in all the following the condition $u \in[0,1]$ is not taken into account. This is permitted by the fact that we consider restrictions of $u$ to smaller sets $\omega$ compactly embedded in $\Omega_{1}$, and therefore we have $u \in[\varepsilon, 1-\varepsilon]$ for some $\varepsilon>0$. We do not recall this in each instance.

\section{On convex functions}

Let us recall some well-known properties of convex functions on $\mathbb{R}^{2}$, and give some definitions. Most of these can be found in classic texts such as [11,12].

We will denote by aff $[p, s]$ the affine function $x \mapsto p \cdot x-s$, where $p \in \mathbb{R}^{2}$ and $s \in \mathbb{R}$ are given and $\cdot$ is the conventional inner product in $\mathbb{R}^{2}$.

A convex function $u$ on $\Omega$ is continuous on $\Omega$, and twice differentiable on a dense subset of $\Omega$. We will denote $\operatorname{dom}(\nabla u)$ the set on which $u$ is once differentiable; in fact, $\nabla u$ is continuous on $\operatorname{dom}(\nabla u)$.

The subdifferential $\partial u$ generalizes the gradient $\nabla u$, and is defined for all $x \in \Omega$ as the set of all slopes of tangent planes at $x$, i.e. $p \in \partial u\left(x_{0}\right)$ if there exists $s \in \mathbb{R}$ such that

$$
\operatorname{aff}[p, s]\left(x_{0}\right)=u\left(x_{0}\right) \quad \text { and } \quad \operatorname{aff}[p, s](x) \leqslant u(x) \quad \forall x .
$$

For every $x \in \Omega, \partial u(x)$ is a closed convex subset of $\mathbb{R}^{2}$, and for $x \in \operatorname{dom}(\nabla u)$ we have $\partial u(x)=\{\nabla u(x)\}$. We will not insist on the difference between a singleton $\{\nabla u(x)\}$ and its value $\nabla u(x)$, and if $A \subset \Omega$, then $\partial u(A)$ is understood to signify $\bigcup_{x \in A} \partial u(x)$. If $A$ is an open convex set, then we have the property $\partial u(A)=\operatorname{conv} \nabla u(A)$ [11, Theorem 25.6].

The second derivative or Hessian matrix $d^{2} u$ and its determinant $\operatorname{det} d^{2} u$ are defined on $\Omega$ as Radon measures [3].

If $p \in \mathbb{R}^{2}$ is given, there exists a unique number $u^{*}(p)$ such that $u \geqslant \operatorname{aff}\left[p, u^{*}(p)\right]$, with equality at least in one point. Indeed we have

$$
u^{*}(p)=\sup _{x \in \Omega}[p \cdot x-u(x)]
$$

which is called the conjugate of $u$. We also have:

$$
u(x)=\max _{p \in \partial u(\Omega)} \operatorname{aff}\left[p, u^{*}(p)\right](x)=\max _{p \in \nabla u(\Omega)} \operatorname{aff}\left[p, u^{*}(p)\right](x)
$$


since $\operatorname{dom}(\nabla u)$ is dense in $\Omega$. It is convenient to introduce an additional notation: if $u$ is differentiable at $x_{0} \in \Omega$, define

$$
\pi\left[u, x_{0}\right]=\operatorname{aff}\left[p, u^{*}(p)\right], \quad \text { with } p=\nabla u\left(x_{0}\right) .
$$

An exposed point of a convex body $C$ is a point $X \in \partial C$ such that there exists a supporting hyperplane through $X$ that only intersects $C$ in $X$ (see [11, p. 162]). An exposed point has the property that a small parallel displacement of the hyperplane leads to a small intersection with $C$. In the context of convex functions $u: \Omega \rightarrow \mathbb{R}$ we shall call $x \in \Omega$ an exposed point if $X=(x, u(x))$ is an exposed point of the graph of $u$. Note that for any convex open subset $\omega \subset \Omega$,

$$
\text { every } x \in \omega \text { is exposed } \Longleftrightarrow u \text { is strictly convex in } \omega .
$$

Applying the conjugation operator twice to a convex function $v$ returns the original function $v: v^{* *}=v$. If $v$ is not convex, then the convex function $v^{* *}$ is called the $\Gamma$-regularization of $v$. It is the largest convex function less than or equal to $v$, or equivalently the supremum of all affine functions less than or equal to $v$.

Most of the functions we deal with in this paper are not defined on $\mathbb{R}^{2}$ but on bounded sets. We implicitly extend such functions by assigning the value $\infty$ outside of the domain of definition. For such functions an alternative convex regularization will be useful, defined by

$$
v^{\#}=\sup \{\pi[v, y]: y \in \operatorname{dom}(\nabla v) \cap \Omega\} .
$$

On $\Omega, v^{\#}$ and $v^{* *}$ coincide; on $\mathbb{R}^{2} \backslash \Omega$, however, if $v \in W^{1, \infty}(\Omega)$, then $v^{\#}$ takes finite values, while $v^{* *}$ equals infinity.

In the proofs that follow we use the following perturbation scheme: for a convex function $u$ and a perturbation $h$ (not necessarily convex), we define

$$
u_{\varepsilon}=u+\varepsilon h .
$$

This function is generally not convex, and we therefore regularize it to give $\widetilde{u}_{\varepsilon}=\left(u_{\varepsilon}\right)^{\#}$. The following lemma states some important properties of this perturbation. Here and throughout this paper a 'measure' will be the Lebesgue measure unless specified otherwise; the Lebesgue measure of a set $\omega$ is denoted $|\omega|$. We also define $\|v\|_{H_{0}^{1}(\Omega)}:=$ $\|\nabla v\|_{L^{2}(\Omega)}$.

Lemma 2. - Let $u: \overline{\Omega^{\prime}} \rightarrow \mathbb{R}$ be strictly convex, and let $h \in W^{1, \infty}\left(\Omega^{\prime}\right)$ have compact support in $\Omega^{\prime}$. Let $\Omega_{c}$ be the union of all open sets in $\Omega^{\prime}$ on which $h$ is convex, and suppose that $\left|\Omega^{\prime} \backslash \Omega_{c}\right|=0$. Set $u_{\varepsilon}=u+\varepsilon h, \widetilde{u}_{\varepsilon}=\left(u_{\varepsilon}\right)^{\#}$, and define $h_{\varepsilon}$ by $\widetilde{u}_{\varepsilon}=u+\varepsilon h_{\varepsilon}$.

(1) The set $\omega_{\varepsilon}=\left\{x \in \Omega^{\prime}: u_{\varepsilon}(x) \neq \widetilde{u}_{\varepsilon}(x)\right\}$ is compactly included in $\Omega^{\prime}$ if $\varepsilon$ is small enough;

(2) $\lim _{\varepsilon \rightarrow 0}\left|\omega_{\varepsilon}\right|=0$;

(3) $\left\|u_{\varepsilon}-\widetilde{u}_{\varepsilon}\right\|_{C_{0}^{0}\left(\Omega^{\prime}\right)}=\mathrm{o}(\varepsilon)$ as $\varepsilon \rightarrow 0$;

(4) $\left\|u_{\varepsilon}-\widetilde{u}_{\varepsilon}\right\|_{H_{0}^{1}\left(\Omega^{\prime}\right)}^{2}=\mathrm{o}(\varepsilon)$ as $\varepsilon \rightarrow 0$. 
Remark. - In part 4 one would expect that the optimal convergence rate is $\mathrm{O}\left(\varepsilon^{2}\right)$ (or possibly $\mathrm{o}\left(\varepsilon^{2}\right)$, in view of 3$)$, but our attemps to prove this have been unsuccessful. A result of this type, however, would simplify the proof of Theorem 1 considerably.

Proof. - To prove part 1, we consider any open set $\Omega_{1}$ such that supp $h \Subset \Omega_{1} \Subset \Omega^{\prime}$. We define

$$
\widetilde{u}=\sup \left\{\pi[u ; y]: y \in \operatorname{dom}(\nabla u) \cap \Omega^{\prime} \backslash \Omega_{1}\right\} .
$$

Since $u$ is strictly convex, we have $u>\widetilde{u}$ in $\Omega_{1}$, hence there exists a number $c>0$ such that $u>\widetilde{u}+c$ in $\operatorname{supp} h$. If $|\varepsilon|<c /\|h\|_{L^{\infty}}$ then $u_{\varepsilon}>\widetilde{u}$ in supp $h$; since $\widetilde{u}$ is a convex function satisfying $\widetilde{u} \leqslant u_{\varepsilon} \leqslant u$ in $\Omega^{\prime}$, we have $\widetilde{u} \leqslant\left(u_{\varepsilon}\right)^{\#} \leqslant u$ in $\Omega^{\prime}$. These inequalities reduce to equalities on $\Omega^{\prime} \backslash \Omega_{1}$, and it follows that $\Omega_{\varepsilon}^{\prime} \subset \Omega_{1}$.

For part 2, pick $x \in \Omega_{c}$ where both $u$ and $h$ are differentiable. We will prove that $x \notin \omega_{\varepsilon}$ if $\varepsilon$ is small enough. The assertion follows from this result by remarking that the characteristic function $\chi_{\omega_{\varepsilon}}$ tends to zero a.e., so that

$$
\left|\omega_{\varepsilon}\right|=\int_{\Omega^{\prime}} \chi_{\omega_{\varepsilon}} \rightarrow 0 \quad \text { as } \varepsilon \rightarrow 0 .
$$

By the strict convexity of $u, x$ is an exposed point; since $\Omega_{c}$ is open there exists $\delta>0$ such that the set

$$
A_{\delta}=\left\{y \in \Omega^{\prime}: u(y)<\pi[u ; x](y)+\delta\right\}
$$

is included in $\Omega_{c}$. Subsequently, if $\varepsilon\|h\|_{L^{\infty}\left(\Omega^{\prime}\right)}<\delta / 2$, then the set

$$
\left\{y \in \Omega^{\prime}: u_{\varepsilon}(y)<\pi[u ; x](y)+\delta / 2\right\}
$$

is included in $A_{\delta}$. Note that since $u_{\varepsilon}$ is strictly convex in $A_{\delta}, \pi\left[u_{\varepsilon} ; x\right]>u_{\varepsilon}$ in $A_{\delta} \backslash\{x\}$.

Since $\nabla u_{\varepsilon}(x) \rightarrow \nabla u(x)$ as $\varepsilon \rightarrow 0$, we can choose $\varepsilon$ so small that

$$
\left\|\pi\left[u_{\varepsilon} ; x\right]-\pi[u ; x]\right\|_{L^{\infty}\left(\Omega^{\prime}\right)}<\frac{\delta}{2} .
$$

Now for all $y \in \Omega^{\prime} \backslash A_{\delta}$,

$$
u_{\varepsilon}(y)-\pi\left[u_{\varepsilon} ; x\right](y)>u_{\varepsilon}(y)-\pi[u, x](y)-\frac{\delta}{2} \geqslant 0,
$$

so that $\pi\left[u_{\varepsilon} ; x\right]>u_{\varepsilon}$ outside $A_{\delta}$. Hence $\widetilde{u}_{\varepsilon}(x)=u_{\varepsilon}(x)$ and we have $x \notin \omega_{\varepsilon}$. This proves part 2.

For part 3 we first show that because of the strict convexity of $u$ there exists a function $\gamma:[0, \infty) \rightarrow \mathbb{R}$, satisfying $\gamma(r)>0$ if $r>0$, such that

$$
u(x) \geqslant u\left(x_{0}\right)+p \cdot\left(x-x_{0}\right)+\gamma\left(\left|x-x_{0}\right|\right),
$$

for all $x, x_{0} \in \Omega^{\prime}$ and all $p \in \partial u\left(x_{0}\right)$. This follows from an argument ad absurdum: if this were not the case, then we can find, using the compactness of $\overline{\Omega^{\prime}}, x, x_{0} \in \overline{\Omega^{\prime}}, x \neq x_{0}$, 
and $p \in \partial u\left(x_{0}\right)$, such that

$$
u(x)=u\left(x_{0}\right)+p \cdot\left(x-x_{0}\right) .
$$

This implies a contradiction with the strict convexity of $u$ in $\overline{\Omega^{\prime}}$. Note that $\gamma(r) \leqslant M r$ for small $r$, for some $M>0$. For convenience we shall also assume that $\gamma$ is monotone increasing and invertible near the origin. This can be achieved without loss of generality.

Suppose that $h_{\varepsilon}(x)-h(x)$ is maximized at $x=x_{0}$. If we write $\widetilde{u}_{\varepsilon}$ as

$$
\widetilde{u}_{\varepsilon}\left(x_{0}\right)=\sup \left\{p \cdot x_{0}+a: p \cdot x+a \leqslant u_{\varepsilon}(x), \forall x \in \Omega^{\prime}\right\},
$$

then we find

$$
\begin{aligned}
& h_{\varepsilon}\left(x_{0}\right)-h\left(x_{0}\right) \\
& \quad=\sup \left\{p \cdot x_{0}+a-\frac{1}{\varepsilon} u\left(x_{0}\right)-h\left(x_{0}\right): \forall x \in \Omega^{\prime}, p \cdot x+a-\frac{1}{\varepsilon} u(x)-h(x) \leqslant 0\right\} .
\end{aligned}
$$

By making the particular choice $\varepsilon p \in \partial u\left(x_{0}\right)$ and applying (11) we find

$$
h_{\varepsilon}\left(x_{0}\right)-h\left(x_{0}\right) \geqslant \sup \left\{a-h\left(x_{0}\right): \forall x \in \Omega^{\prime}, a-\frac{1}{\varepsilon} \gamma\left(\left|x-x_{0}\right|\right)-h(x) \leqslant 0\right\} \text {. }
$$

If the inequality in the conditional part is saturated at $x=x_{1}$, i.e. $a-\gamma\left(\left|x_{1}-x_{0}\right|\right) / \varepsilon-$ $h\left(x_{1}\right)=0$, then

$$
\gamma\left(\left|x_{1}-x_{0}\right|\right)=\varepsilon\left(h\left(x_{0}\right)-h\left(x_{1}\right)\right),
$$

so that $x_{1}-x_{0} \leqslant \gamma^{-1}\left(\varepsilon\|h\|_{L^{\infty}}\right)$; by re-inserting this in the equation above we find that $\gamma\left(\left|x_{1}-x_{0}\right|\right) \leqslant \varepsilon\|h\|_{W^{1, \infty}} \gamma^{-1}\left(\varepsilon\|h\|_{L^{\infty}}\right)$ and thus

$$
0 \geqslant h_{\varepsilon}\left(x_{0}\right)-h\left(x_{0}\right) \geqslant \frac{1}{\varepsilon} \gamma\left(\left|x_{1}-x_{0}\right|\right)+h\left(x_{1}\right)-h\left(x_{0}\right) \leqslant C \gamma^{-1}\left(\varepsilon\|h\|_{L^{\infty}}\right) .
$$

Since $h_{\varepsilon}-h$ is supposed to be maximal at $x=x_{0}$, we therefore have

$$
\left\|u_{\varepsilon}-\widetilde{u}_{\varepsilon}\right\|_{C_{0}^{0}\left(\Omega^{\prime}\right)}=\varepsilon\left\|h_{\varepsilon}-h\right\|_{C_{0}^{0}\left(\Omega^{\prime}\right)}=\mathrm{o}(\varepsilon) \quad \text { as } \varepsilon \rightarrow 0 .
$$

For part 4 , we note that since $\Delta u_{\varepsilon}$ and $\Delta \tilde{u}_{\varepsilon}$ are bounded in the space of Radon measures $R M\left(\Omega^{\prime}\right)$, we therefore have

$$
\begin{aligned}
\left\|\tilde{u}_{\varepsilon}-u_{\varepsilon}\right\|_{H^{1}\left(\Omega^{\prime}\right)}^{2} & =-\int\left(\widetilde{u}_{\varepsilon}-u_{\varepsilon}\right) \Delta\left(\widetilde{u}_{\varepsilon}-u_{\varepsilon}\right) \\
& \leqslant\left\|\tilde{u}_{\varepsilon}-u_{\varepsilon}\right\|_{C_{0}^{0}\left(\Omega^{\prime}\right)}\left\|\Delta\left(\tilde{u}_{\varepsilon}-u_{\varepsilon}\right)\right\|_{R M\left(\Omega^{\prime}\right)}
\end{aligned}
$$

and this last term is of order $o(\varepsilon)$.

\section{Regularity of the minimizers}

We turn to the study of properties of the minimizers of $F$. If $u$ is convex, the set of limits of $\partial u(x)$ as $x \rightarrow x_{0}$ (or, equivalently, of $\nabla u(x)$ for those $x$ for which this is 
defined) is the boundary of the subdifferential $\partial u\left(x_{0}\right)$ [11, Corollary 25.4.1]. We will denote this boundary $\partial\left[\partial u\left(x_{0}\right)\right]$. As mentioned above, the subdifferential is a singleton if and only if $u$ is differentiable at $x_{0}$.

For given $x_{0} \in \Omega$ we define polar coordinates with center $x_{0}$, that is $r=\left|x-x_{0}\right|$, $\phi=\arg \left(x-x_{0}\right)$, so that $u(x)=u\left(x_{0}\right)+r g(\phi)+\mathrm{o}(r)$ for some continuous function $g: S^{1} \rightarrow \mathbb{R}$. Denoting $\left(e_{r}, e_{\phi}\right)$ the varying orthonormal basis at $x \neq x_{0}$ (such that $\left.e_{r}=\left(x-x_{0}\right) / r\right)$, we define $V(\phi)$ as the limit of $\nabla u\left(x_{0}+t e_{r}(\phi)\right)$, as $t>0$ goes to zero $\left(\phi \in S^{1}\right.$ fixed). Expressed differently, $V(\phi)$ is the gradient at $x_{0}+t e_{r}(\phi)$, for any $t>0$, of the convex function $\hat{u}(x):=u\left(x_{0}\right)+r g(\phi)$ whose graph is the tangent cone of the graph of $u$ at $x_{0}$ (note that $\hat{u} \leqslant u$ by the convexity of $u$ ). Therefore we have $V(\phi)=g(\phi) e_{r}(\phi)+g^{\prime}(\phi) e_{\phi}(\phi)$. A little calculation indicates that the convexity of $\hat{u}$ is caracterized by the property $g+g^{\prime \prime} \geqslant 0$ in the sense of distributions on $S^{1}$.

The map $\phi \in S^{1} \mapsto V(\phi)$ is a partial parametrization of $\partial\left[\partial u\left(x_{0}\right)\right]$ (the parametrization is incomplete wherever $V$ is discontinuous, and non-injective whenever $V$ is constant). The function $V$ is constant if and only if $u$ is differentiable at $x_{0}$.

We first derive a simple consequence of the minimisation.

LEMMA 3. - Let $u$ be a minimizer of (4), and suppose that $\partial u\left(x_{0}\right)$ has non-empty interior. We define the function $g$ as described above. Let $H_{g} \subset C^{0}\left(S^{1}\right)$ be the set of functions $h$ satisfying $h \leqslant g$ and $h+h^{\prime \prime} \geqslant 0$ in distributional sense. Then

$$
\forall h \in H_{g}, \quad 0 \leqslant \int_{S^{1}} \frac{f\left(h e_{r}+h^{\prime} e_{\phi}\right)-f\left(g e_{r}+g^{\prime} e_{\phi}\right)}{(g-h)^{2}} .
$$

Moreover, if $V$ is discontinuous at $\phi_{0} \in S^{1}$, with left and right limits $V_{1}:=V\left(\phi_{0}-\right)$, $V_{2}:=V\left(\phi_{0}+\right)$, then

$$
\forall t \in[0,1], \quad f\left(t V_{1}+(1-t) V_{2}\right) \geqslant t f\left(V_{1}\right)+(1-t) f\left(V_{2}\right) .
$$

Proof. - We prove (12) only for $h<g$; the more general case follows from a density argument, taking into account that, since $\partial u\left(x_{0}\right)$ has non-empty interior, there exists a dense subset of such functions $h$ in $H_{g}$.

Let us choose $h \in H_{g}, h<g$; we define $\hat{v}(x):=u\left(x_{0}\right)+r h(\phi)$ in polar coordinates. Since $h+h^{\prime \prime} \geqslant 0, \hat{v}$ is convex, and $\hat{v}(x)<\hat{u}(x) \leqslant u(x)$ for all $x \neq x_{0}$.

Therefore, for any $\varepsilon>0$ small enough, the function $v:=\max (u, \hat{v}+\varepsilon)$ is convex, and coincides with $u$ except in a small neighbourhood of $x$, a set $\{r<\sigma(\varepsilon, \phi)\}$ in polar coordinates; inside this set, we have $\nabla v=\nabla \hat{v}=h e_{r}+h^{\prime} e_{\phi}$. Note that $\sigma(\varepsilon, \phi)=$ $\varepsilon /(g(\phi)-h(\phi))+\mathrm{o}(\varepsilon)$ as $\varepsilon \rightarrow 0$. Also, since $u\left(x_{0}\right) \in(0,1)$ by assumption, we have $v(x) \in[0,1]$ for all $x$ if $\varepsilon>0$ is small enough. Hence $F(v) \geqslant F(u)$, that is

$$
\begin{aligned}
0 & \leqslant \int_{S^{1}} \int_{0}^{\sigma(\varepsilon, \phi)}\left[f\left(h e_{r}+h^{\prime} e_{\phi}\right)-f(\nabla u(r, \phi))\right] r d r d \phi \\
& \leqslant \varepsilon^{2} \int_{S^{1}}^{\frac{1}{s(\phi)-h(\phi)}} \int_{0}^{1}\left[f\left(h e_{r}+h^{\prime} e_{\phi}\right)-f(\nabla u(\varepsilon s, \phi))\right] s d s d \phi+\mathrm{o}\left(\varepsilon^{2}\right)
\end{aligned}
$$


using the change of variable $r:=\varepsilon s$. Since this holds for any $\varepsilon>0$ small enough, we can divide by $\varepsilon^{2}$ and let $\varepsilon$ tend to 0 ; the term $\nabla u(\varepsilon s, \phi)$ converges to $V(\phi)=g e_{r}+g^{\prime} e_{\phi}$ and $f\left(h e_{r}+h^{\prime} e_{\phi}\right)$ does not change. We integrate $s d s$ to obtain (12).

We now turn to the proof of (13). We write $\gamma^{+}:=g^{\prime}\left(\phi_{0}+\right)$ and $\gamma^{-}:=g^{\prime}\left(\phi_{0}-\right)$ for the right and left derivative of $g$ at $\phi_{0}$, and consider any number $a \in\left(\gamma^{-}, \gamma^{+}\right)$; let $V_{a}:=g\left(\phi_{0}\right) e_{r}+a e_{\phi}\left(\phi_{0}\right)$, and $g_{a}(\phi):=g\left(\phi_{0}\right) \cos \left(\phi-\phi_{0}\right)+a \sin \left(\phi-\phi_{0}\right)$. The convex function $v_{a}(r, \phi):=u\left(x_{0}\right)+r g_{a}(\phi)$ is in fact an affine function (since $g_{a}+g_{a}^{\prime \prime}=0$ ) with gradient $V_{a}$; moreover, by the choice of $a, g_{a}(\phi)<g(\phi)$ for any $\phi \neq \phi_{0}$, or equivalently, $\hat{u}(x)>v_{a}(x)$ for any $x \notin x_{0}+\mathbb{R}_{+} e_{r}\left(\phi_{0}\right)$.

We pick $\varepsilon>0$ and define

$$
h:=\max \left((1-\varepsilon) g_{a}, \frac{g}{2}\right) .
$$

There exist $\alpha_{\varepsilon}, \beta_{\varepsilon} \in S^{1}$ such that $h=(1-\varepsilon) g_{a}$ in $\left(\alpha_{\varepsilon}, \beta_{\varepsilon}\right)$, and $h=g / 2$ in $\left(\beta_{\varepsilon}, \alpha_{\varepsilon}\right)$. We have $\lim _{\varepsilon \rightarrow 0} \alpha_{\varepsilon} \neq \phi_{0} \neq \lim _{\varepsilon \rightarrow 0} \beta_{\varepsilon}$.

Let us now apply (12) to $h$. The function

$$
\delta(\phi):=\frac{f\left(h e_{r}+h^{\prime} e_{\phi}\right)-f\left(g e_{r}+g^{\prime} e_{\phi}\right)}{(g-h)^{2}}
$$

becomes infinite near $\phi_{0}$ as $\varepsilon \rightarrow 0$; more precisely, for $\phi \in\left(\phi_{0}, \beta_{\varepsilon}\right)$ we have

$$
\delta(\phi)=\frac{f\left((1-\varepsilon) V_{a}\right)-f\left(g e_{r}+g^{\prime} e_{\phi}\right)}{\left(g-g_{a}+\varepsilon g_{a}\right)^{2}} .
$$

To estimate this term for small $\varepsilon$ we note that if $a, b$, and $c$ are continuous functions and $b$ is continuously differentiable, satisfying $b(0)=0, b^{\prime}(0) \neq 0$, and $c(0) \neq 0$, then

$$
\int_{0}^{1} \frac{a(x) d x}{(b(x)+\varepsilon c(x))^{2}}=\frac{a(0)}{\varepsilon b^{\prime}(0) c(0)}+\mathrm{o}(1 / \varepsilon) \quad \text { as } \varepsilon \rightarrow 0 .
$$

Hence, for small $\varepsilon$ :

$$
\int_{\phi_{0}}^{\beta_{\varepsilon}} \delta=\frac{1}{\varepsilon} \frac{\left(f\left(V_{a}\right)-f\left(V_{2}\right)\right)}{g\left(\phi_{0}\right)\left(\gamma^{+}-a\right)}+\mathrm{o}(1 / \varepsilon) .
$$

Similarly,

$$
\int_{\alpha_{\varepsilon}}^{\phi_{0}} \delta=-\frac{1}{\varepsilon} \frac{\left(f\left(V_{a}\right)-f\left(V_{1}\right)\right)}{g\left(\phi_{0}\right)\left(\gamma^{-}-a\right)}+\mathrm{o}(1 / \varepsilon) .
$$

We recall that $\int_{S^{1}} \delta \geqslant 0$, hence in particular the dominant terms must have nonnegative sum. This gives:

$$
\frac{f\left(V_{a}\right)-f\left(V_{2}\right)}{\left(\gamma^{+}-a\right)} \geqslant \frac{f\left(V_{a}\right)-f\left(V_{1}\right)}{\left(\gamma^{-}-a\right)},
$$


which is equivalent to (13) for an appropriate choice of $t$. By varying $a$ the result follows for all $t \in[0,1]$.

We continue by studying the character of $V$ in a particular case. The following technical lemma uses techniques similar to the previous ones, but will be needed only in Section 5. Note that the set $N_{0}$ is defined in (6).

LEMMA 4. - Let $u$ be a minimizer of $F$, and suppose that $\operatorname{div} d f(\nabla u)=0$ (in $a$ distributional sense) on a subset $\Omega^{\prime} \subset \Omega \backslash N_{0}$ where $u$ is stricly convex. Assume that $\partial u\left(x_{0}\right)$ has non-empty interior for some $x_{0} \in \Omega^{\prime}$, and that there exists a neighborhood $Q$ of $\partial u\left(x_{0}\right)$ such that $f$ has a strictly positive eigenvalue (and a strictly negative one, since $f$ is nowhere convex) and

$$
\bigcap_{p \in Q}\left\{\xi \in \mathbb{R}^{2} ; d^{2} f(p) \xi \cdot \xi<0\right\} \neq \emptyset
$$

Then the limit gradient field $V$ has at least two discontinuities in $S^{1}$. Moreover, at every discontinuity we have not only (13), but also

$$
\left[d f\left(V_{1}\right)-d f\left(V_{2}\right)\right] \cdot\left(V_{1}-V_{2}\right)=0 \text {. }
$$

Note that the condition (14) is satisfied if the diameter of $\partial u\left(x_{0}\right)$ is small enough.

Proof. - By the hypothesis, for all $p \in Q$, there exist linearly independent vectors $q_{1}(p), q_{2}(p)$ of unit length, depending smoothly on $p$, such that $d^{2} f(p) \cdot q_{i}(p) \cdot q_{i}(p)=$ 0 for $i=1,2$. By (14), there exist two closed convex cones $K_{i} \subset \mathbb{R}^{2}$ such that $q_{i}(Q) \subset$ $K_{i}$ and $K_{1} \cap K_{2}=K_{1} \cap\left(-K_{2}\right)=\{0\}$.

We fix a point $x_{0}$ as the origin of coordinates; subtracting an affine function from $u$ (and translating $f$ ), we may assume that $u(0)=0$, and that $u$ attains a minimum at $x=0$ (this is a strict minimum since $u$ is strictly convex). We will use polar coordinates with respect to the center $0: r:=|x|, \phi:=\arg x \in S^{1}$. As in the proof of Lemma 3, $\left(e_{r}(\phi), e_{\phi}(\phi)\right)$ is a local orthonormal basis, and $V(\phi)$ is the limit, as $r \rightarrow 0$ of $\nabla u\left(r e_{r}\right)$; this limit is not constant with respect to $\phi$ (since otherwise $u$ would be differentiable at 0 [11, Theorem 25.2]). We have $V(\phi)=g(\phi) e_{r}+g^{\prime}(\phi) e_{\phi}$.

By a blow-up argument very similar to that used in the proof of Lemma 3 it follows that the function $\hat{u}(x):=u\left(x_{0}\right)+r g(\phi)$ again satisfies $(27)$, i.e.

$$
\operatorname{div} d f(\nabla \hat{u})=0
$$

in a distributional (or measure-) sense in $\mathbb{R}^{2}$. For any $\phi \in S^{1}$ such that $V$ is continuous at $\phi$, this reduces to

$$
d^{2} f(V(\phi)) \cdot V^{\prime}(\phi) \cdot e_{\phi}=0
$$

and if $V$ has a discontinuity at $\phi$, then

$$
[d f(V(\phi+))-d f(V(\phi-))] \cdot(V(\phi+)-V(\phi-))=0 .
$$

This last equation gives (15). 
First suppose that $V$ is a continuous function of $\phi$ on some connected part $J \subset S^{1}$ having nonempty interior, implying by convexity that $V, g^{\prime} \in W^{1,1}(J)$. We claim that $V$ is constant in $J$. Indeed, using $V^{\prime}=\left(g+g^{\prime \prime}\right) e_{\phi}$, we have

$$
\left(g(\phi)+g^{\prime \prime}(\phi)\right) d^{2} f(V(\phi)) \cdot e_{\phi} \cdot e_{\phi}=0 \quad \text { for a.e. } \phi \in J
$$

Therefore, for almost all $\phi \in J$, either $V^{\prime}(\phi)=0$, or $e_{\phi} \in\left\{ \pm q_{1}(V(\phi)), \pm q_{2}(V(\phi))\right\}$. We can write $J$ as a disjoint union

$$
J=A_{1}^{+} \cup A_{1}^{-} \cup A_{2}^{+} \cup A_{2}^{-} \cup B,
$$

such that $e_{\phi}= \pm q_{i}(V(\phi))$ in $J$ if and only if $\phi \in A_{i}^{ \pm}$. Note that by continuity the sets $A_{i}^{ \pm}$are closed in $J$.

The sets $A_{i}^{ \pm}$are connected parts of $J$ (it is convenient to write them as intervals). Indeed, if $\phi_{1}, \phi_{2} \in A_{1}^{+}$, then

$$
\begin{aligned}
\text { either } & {\left[\phi_{1}, \phi_{2}\right] \cap A_{1}^{-}=\left[\phi_{1}, \phi_{2}\right] \cap A_{2}^{ \pm}=\emptyset } \\
\text { or } & {\left[\phi_{2}, \phi_{1}\right] \cap A_{1}^{-}=\left[\phi_{2}, \phi_{1}\right] \cap A_{2}^{ \pm}=\emptyset }
\end{aligned}
$$

(note that both $\left[\phi_{1}, \phi_{2}\right]$ and $\left[\phi_{2}, \phi_{1}\right]$ are intervals in $S^{1}$ ). This follows from the monotonicity of $e_{\phi}$ and the disjoint nature of the cones $K_{i}$. Now, without loss of generality, suppose that the first of (18) is true. Since $A_{1}^{+}$is closed, if $B \cap\left[\phi_{1}, \phi_{2}\right] \neq \emptyset$, then there exist $\phi_{3}, \phi_{4} \in A_{1}^{+}$, with $\left[\phi_{3}, \phi_{4}\right] \subset\left(\phi_{1}, \phi_{2}\right)$, such that $\left(\phi_{3}, \phi_{4}\right) \subset B$. On $\left(\phi_{3}, \phi_{4}\right), e_{\phi} \notin\left\{ \pm q_{1}(V(\phi)), \pm q_{2}(V(\phi))\right\}$ and therefore $V^{\prime} \equiv 0$, so that $q_{1}\left(V\left(\phi_{3}\right)\right)=$ $q_{1}\left(V\left(\phi_{4}\right)\right)$. But since $\phi_{3} \neq \phi_{4}$, we have $e_{\phi_{3}} \neq e_{\phi_{4}}$, which contradicts the assumption that $\phi_{3}, \phi_{4} \in A_{1}^{+}$. Therefore $A_{1}^{+}$, and in a similar manner the other $A_{i}^{ \pm}$, are connected.

Let us also observe that some of these sets can have empty interior; if they all do, then $V^{\prime}=0$ a.e. in $J$, and $V$ is constant, and our claim is proved. So, assume that $J \backslash B$ has non-empty interior; we will prove a contradiction.

We note that $g+g^{\prime \prime}>0$ in the interior of every interval $A_{i}^{ \pm}$. Indeed, if we assume for instance that $A_{1}^{+}$has non-empty interior, we have here $e_{\phi}=+q_{1}(V(\phi))$ for all $\phi \in A_{1}^{+}$, from its definition, and therefore,

$$
\begin{aligned}
1 & =e_{\phi} \wedge \frac{d}{d \phi} e_{\phi}=e_{\phi} \wedge \frac{d}{d \phi} q_{1}(V(\phi)) \\
& =e_{\phi} \wedge \nabla q_{1}(V(\phi)) \cdot V^{\prime}(\phi)=\left(g+g^{\prime \prime}\right) e_{\phi} \wedge \nabla q_{1}(V(\phi)) \cdot e_{\phi},
\end{aligned}
$$

implying that $g+g^{\prime \prime}>$ const. $>0$.

Let $h: S^{1} \rightarrow \mathbb{R}$ be a function in $H_{g}$, as defined in Lemma 3. In particular, we can choose $h$ in the form $h:=(1-\varepsilon \sigma) g$, with $\varepsilon>0$ small and $\sigma \in C^{2}\left(S_{1}\right), \sigma \geqslant 0$, having a derivative with compact support in the interior of $J \backslash B$; since the derivatives of $\sigma$ are bounded, and $g+g^{\prime \prime}>0$ in the interior of $J \backslash B$, we have $h+h^{\prime \prime} \geqslant 0$ in $S^{1}$ for small enough $\varepsilon$.

Developing (12) with respect to $\varepsilon$ yields

$$
\int_{S^{1}} d^{2} f(V(\phi))\left(e_{r}+k(\phi) e_{\phi}\right) \cdot\left(e_{r}+k(\phi) e_{\phi}\right) d \phi \geqslant 0
$$


with

$$
k(\phi):=\frac{(\sigma g)^{\prime}}{\sigma g}(\phi)=\frac{\sigma^{\prime}}{\sigma}(\phi)+\frac{g^{\prime}}{g}(\phi)
$$

(the first-order term vanishes by (16)). Recall that $d^{2} f(V(\phi)) e_{\phi} \cdot e_{\phi}=0$ in the interior of $J \backslash B$; hence (19) implies

$$
\begin{aligned}
& 2 \int_{J \backslash B} d^{2} f(V(\phi)) e_{\phi} \cdot e_{r} \frac{\sigma^{\prime}}{\sigma} d \phi \geqslant I_{g}, \\
& I_{g}:=-\int_{S^{1}} d^{2} f(V(\phi))\left(e_{r}+\frac{g^{\prime}}{g} e_{\phi}\right) \cdot\left(e_{r}+\frac{g^{\prime}}{g} e_{\phi}\right) d \phi .
\end{aligned}
$$

Note that the right-hand side $I_{g}$ does not depend on $\sigma$. We choose $\sigma^{\prime} / \sigma$ close to the sum of two Dirac measures, by considering a $\sigma$ close to a step function (we cannot have only one Dirac, by the periodicity of $\sigma)$; it follows that $d^{2} f(V(\phi)) e_{\phi} \cdot e_{r} \equiv c=$ const in the interior of $J \backslash B$. Taking $d^{2} f(V(\phi)) e_{\phi} \cdot e_{\phi}=0$ into account, we get $d^{2} f(V) e_{\phi}=c e_{r}$ in $J \backslash B$ (note that although $J \backslash B$ is not connected, the constant $c$ is the same for each connected part).

For any function $k \in C^{2}\left(S^{1}\right)$, a short computation gives

$$
\left[d f(V(\phi)) \cdot\left(k e_{r}+k^{\prime} e_{\phi}\right)\right]^{\prime}=c k\left(g+g^{\prime \prime}\right)+\left(k+k^{\prime \prime}\right) d f(V) \cdot e_{\phi}
$$

in $J \backslash B$, and also in $B$ where $g+g^{\prime \prime}=0$. Since the variation of $V$ is assumed to be small, $d f(V) \cdot e_{\phi}$ must change sign in $S^{1}$, and hence we can choose a function $k \geqslant 0$ such that the sign of $k+k^{\prime \prime}$ coincides with the sign of $d f(V) \cdot e_{\phi}$. This choice of $k$ renders the right-hand side of the last equation nonnegative, contradicting the periodicity of $d f(V(\phi)) \cdot\left(k e_{r}+k^{\prime} e_{\phi}\right)$.

This ends the proof of our claim, that if $V$ is continuous on any connected part $J \subset S^{1}$, then it is just constant on $J$.

As a consequence, and since by assumption $V$ is not constant in $S^{1}$, we conclude that $V$ is not continuous, and has at least two discontinuities in $S^{1}$.

\section{Proof of Theorem 1}

The proof of the main theorem of this paper, Theorem 1, is based on the following instability result. It conveys the property that a minimizer can not be a stationary point and strictly convex at the same time.

LEMMA 5. - Let $u$ minimize $F$ and suppose that $u$ is strictly convex in a convex open subset $\Omega_{1} \subset \Omega$. Then there exists a convex polyhedral function $\theta: \bar{\Omega} \rightarrow \mathbb{R}$ such that

$$
\omega_{\theta}=\{x \in \Omega: u(x)<\theta(x)\}
$$

is non-empty and included in $\Omega_{1}$, and such that

$$
\int_{\omega_{\theta}} d f(\nabla u) \nabla(u-\theta)<0 .
$$


A convex polyhedral function is the pointwise supremum of a finite number of affine functions.

We defer the proof of this lemma to the next section, and continue first with the proof of Theorem 1, assuming for the moment that this lemma is proved.

Suppose that $u$ is strictly convex in $\Omega_{1}$, and let $\theta$ be the polyhedral function given by Lemma 5. We write simply $\omega$ for the corresponding set $\omega_{\theta}$. Set $\phi:=u-\theta$ in $\bar{\omega}$, and extend $\phi$ outside of $\bar{\omega}$ by zero. Define for $\varepsilon>0$ the functions

$$
u_{\varepsilon}=u+\varepsilon \phi \quad \text { and } \quad \widetilde{u}_{\varepsilon}=\left(u_{\varepsilon}\right)^{\#} .
$$

Note that if $\varepsilon$ is small, then by Lemma 2 the set

$$
\widetilde{\omega}_{\varepsilon}=\left\{x \in \Omega ; \widetilde{u}_{\varepsilon}(x)<u_{\varepsilon}(x)\right\}
$$

is compactly included in $\Omega_{1}$, and the Lebesgue measure of $\widetilde{\omega}_{\varepsilon}$ tends to zero as $\varepsilon \rightarrow 0$. Therefore $\widetilde{u}_{\varepsilon}$ is admissible if $\varepsilon$ is small enough.

We find on applying a Taylor series expansion to $F$ at $u$,

$$
F\left(\widetilde{u}_{\varepsilon}\right)-F(u)=d F(u)\left(\widetilde{u}_{\varepsilon}-u\right)+\mathrm{O}\left(\left\|\nabla\left(\widetilde{u}_{\varepsilon}-u\right)\right\|_{L^{2}(\Omega)}^{2}\right) .
$$

The last term, $\mathrm{O}\left(\left\|\nabla\left(\widetilde{u}_{\varepsilon}-u\right)\right\|_{L^{2}(\Omega)}^{2}\right)$, is of order $\mathrm{o}(\varepsilon)$ by Lemma 2, part 4.

The first term on the right-hand side of (21) is split into two parts:

$$
\begin{aligned}
d F(u)\left(\widetilde{u}_{\varepsilon}-u\right) & =d F(u)\left(\widetilde{u}_{\varepsilon}-u_{\varepsilon}\right)+d F(u)\left(u_{\varepsilon}-u\right) \\
& =d F(u)\left(\widetilde{u}_{\varepsilon}-u_{\varepsilon}\right)+\varepsilon d F(u) \phi .
\end{aligned}
$$

The second of these two is of order $\varepsilon$ since by the choice of $\theta$ we have $d F(u) \phi<0$.

To estimate the first term we note that

$$
\begin{aligned}
\left|d F(u)\left(\widetilde{u}_{\varepsilon}-u_{\varepsilon}\right)\right| & =\left|\int_{\Omega} d f(\nabla u) \nabla\left(\widetilde{u}_{\varepsilon}-u_{\varepsilon}\right)\right| \\
& =\left|\int_{\Omega}\left(\widetilde{u}_{\varepsilon}-u_{\varepsilon}\right) \operatorname{div} d f(\nabla u)\right| \\
& \leqslant M\left\|d^{2} u\right\|_{R M(\Omega)}\left\|\widetilde{u}_{\varepsilon}-u_{\varepsilon}\right\|_{C_{0}^{0}(\Omega)},
\end{aligned}
$$

where $M$ is a bound on the second derivative $d^{2} f$. Therefore we have

$$
d F(u)\left(\widetilde{u}_{\varepsilon}-u_{\varepsilon}\right)=\mathrm{o}(\varepsilon),
$$

using Lemma 2, part 3.

By combining estimates (21) and (23) with (22) we find

$$
F\left(\widetilde{u}_{\varepsilon}\right)-F(u) \leqslant-c \varepsilon
$$

for some $c>0$, which contradicts the minimality of $u$. 


\section{The instability lemma}

We continue with the proof of Lemma 5, which we repeat here for convenience:

LEMMA 5. - Let u minimize $F$ and suppose that $u$ is strictly convex in a convex open subset $\Omega_{1} \subset \Omega$. Then there exists a convex polyhedral function $\theta: \bar{\Omega} \rightarrow \mathbb{R}$ such that

$$
\omega_{\theta}=\{x \in \Omega: u(x)<\theta(x)\}
$$

is non-empty and included in $\Omega_{1}$, and such that

$$
\int_{\omega_{\theta}} d f(\nabla u) \nabla(u-\theta)<0 .
$$

The proof of this lemma relies strongly on a simple density property of the vector space generated by the 'caps' of a given strictly convex function, which can be expressed as follows:

LemmA 6. - Let $\Omega_{1} \subset \mathbb{R}^{2}$ be a convex domain, $u: \Omega_{1} \rightarrow \mathbb{R}$ be strictly convex. Let $\Theta$ be the set of all convex polyhedral functions $\theta$ such that the set $\omega_{\theta}$ defined by (24) is non-empty and included in $\Omega_{1}$; for each $\theta \in \Theta$, we consider the 'cap' function $\gamma_{\theta}:=(\theta-u)_{+} \in C_{0}^{0}\left(\overline{\Omega_{1}}\right)$ (the set of continuous functions vanishing on the boundary). Then the vector field generated by these functions is dense in $C_{0}^{0}\left(\overline{\Omega_{1}}\right)$ (in the usual $C^{0}$ topology).

Proof. - Note that $\Theta$ is not empty since $\Omega_{1}$ consists of exposed points of $u$ by (10). Therefore, if $x_{0} \in \Omega_{1} \cap \operatorname{dom}(\nabla u)$ is given, any affine function $\pi\left[u ; x_{0}\right]+\varepsilon$ is in $\Theta$, if $\varepsilon>0$ is small enough.

We denote $E:=\operatorname{Vect}\left\{\gamma_{\theta}\right\}_{\theta \in \Theta}$ the (algebraic) vector field generated by $\Theta$, and $\bar{E}$ its closure. We have to prove that $\bar{E}=C_{0}^{0}\left(\overline{\Omega_{1}}\right)$; in order to do that, it suffices to prove that $\bar{E}$ is a lattice and separates points, in view of the Kakutani-Krein theorem (see for instance [10, p. 104]). We recall that a lattice is a subset of $C^{0}$ that is stable under the min and max operations.

If $\theta_{1}, \theta_{2}$ are given in $\Theta$, then $\theta_{3}:=\max \left(\theta_{1}, \theta_{2}\right)$ is a convex polyhedral function; moreover, $\omega_{\theta_{3}}=\omega_{\theta_{1}} \cup \omega_{\theta_{2}}$ is non-empty and included in $\Omega_{1}$, so that $\theta_{3} \in \Theta$. In addition $\theta_{3} \geqslant \theta_{1}$, so that $\omega_{\theta_{3}}$ contains $\omega_{\theta_{1}}$ and therefore is not empty; this implies that $\theta_{3} \in \Theta$. We now have

$$
\gamma_{\theta_{3}}=\max \left(0, \theta_{3}-u\right)=\max \left(0, \max \left(\theta_{1}-u, \theta_{2}-u\right)\right)=\max \left(\gamma_{\theta_{1}}, \gamma_{\theta_{2}}\right) .
$$

Hence $E$ is stable under the max operation, and therefore $\bar{E}$ is also stable. On the other hand, if $f, g \in \bar{E}$ are given, then $\min (f, g)=f+g-\max (f, g)$ belongs to $\bar{E}$. This proves that $\bar{E}$ is a lattice.

It is easy to see that $\bar{E}$ separates points; indeed, even the smaller subset

$$
\left\{\gamma_{\theta} ; \theta \in \Theta \text { affine }\right\}
$$

separates points since $u$ is strictly convex. 
Proof of Lemma 5. - The proof proceeds in five steps.

Step 1 . For every $\theta \in \Theta, \int_{\omega_{\theta}} d f(\nabla u) \nabla(u-\theta) \leqslant 0$.

Indeed, suppose that $\int_{\omega_{\theta}} d f(\nabla u) \nabla(u-\theta)>0$ for some $\theta$. Define $\phi=u-\theta$ on $\omega_{\theta}$, and extend $\phi$ by zero outside of $\omega_{\theta}$. The function

$$
u_{\varepsilon}=u-\varepsilon \phi
$$

is admissible if $0 \leqslant \varepsilon \leqslant 1$. Using the Taylor expansion

$$
f(p)=f\left(p_{0}\right)+d f\left(p_{0}\right)\left(p-p_{0}\right)+g\left(p_{0}, p\right)\left(p-p_{0}\right) \cdot\left(p-p_{0}\right),
$$

where $g: \mathbb{R}^{2} \times \mathbb{R}^{2} \rightarrow \mathbb{R}^{2 \times 2}$ is bounded on compact subsets, we find

$$
F\left(u_{\varepsilon}\right)-F(u)=-\varepsilon \int_{\omega_{\theta}} d f(\nabla u) \nabla \phi+\varepsilon^{2} \int_{\omega_{\theta}} g\left(\nabla u, \nabla u_{\varepsilon}\right) \nabla \phi \cdot \nabla \phi .
$$

Clearly this contradicts the minimality of $u$ when $\varepsilon$ is small.

Step 2. If

$$
\int_{\omega_{\theta}} d f(\nabla u) \nabla(u-\theta)=0, \quad \forall \theta \in \Theta
$$

then

$$
\operatorname{div} d f(\nabla u) \equiv 0 \quad \text { in } \Omega_{1} \text {, in the sense of measures. }
$$

Indeed, if we use the notations of Lemma 6, then Eq. (26) can be written $\int_{\Omega_{1}} d f(\nabla u) \times$ $\nabla \gamma_{\theta}=0, \forall \theta \in \Theta$. Therefore, the continuous linear map $\varphi \mapsto \int_{\Omega_{1}} d f(\nabla u) \nabla \varphi$ vanishes identically on $E$, and therefore on $\bar{E}=C_{0}^{0}\left(\Omega_{1}\right)$. This implies (27).

Step 3. We prove now that there exists an open subset $\Omega_{2} \subset \Omega_{1}$, such that for any $x \in \Omega_{2}, \partial u(x)$ has empty interior.

We argue by contradiction and assume that there exists a dense set $S \subset \Omega_{1}$ of points $x$ where $\partial u(x)$ has non-empty interior. In particular, if we consider some point $y \in \operatorname{dom}(\nabla u) \cap \Omega_{1}$, there exists a sequence $\left(x^{n}\right) \subset S$ with limit $y$. Since we assume that $u$ is differentiable at $y$, the diameter of $\partial u\left(x^{n}\right)$ tends to 0 as $n \rightarrow \infty$ [11, Corollary 24.5.1]; more precisely,

$$
\lim _{n \rightarrow \infty} \sup _{p \in \partial u\left(x^{n}\right)}|\nabla u(y)-p|=0 .
$$

We have mainly to consider the case where $y$ is such that $d^{2} f(\nabla u(y))$ has two nonzero eigenvalues, with different signs. For if not, since we assume that $f$ is nowhere convex, both eigenvalues of $d^{2} f(\nabla u(y))$ are non-positive at every point, that is, $f$ is concave on $\nabla u\left(\Omega_{1}\right)$; in that case, it is clear that $u$ cannot be stricly convex.

As a consequence, there exists an open neigborhood $Q \subset \mathbb{R}^{2}$ of $\nabla u(y)$, such that for each $p \in Q$, the matrix $d^{2} f(p)$ has one strictly positive and one strictly negative eigenvalue. Hence, for all $p \in Q$, there exist linearly independent vectors $q_{1}(p), q_{2}(p)$ of unit length, depending smoothly on $p$, such that $d^{2} f(p) \cdot q_{i}(p) \cdot q_{i}(p)=0$ for $i=1,2$. From (28), we can assume that for every $n, \partial u\left(x^{n}\right) \subset Q$. 
For every $n$, we are now in the conditions of Lemmas 3 and 4 at $x^{n}$. Hence there exist $V_{1}^{n}, V_{2}^{n}$ in $\partial u\left(x^{n}\right)$ such that

$$
\begin{aligned}
& {\left[d f\left(V_{1}^{n}\right)-d f\left(V_{2}^{n}\right)\right] \cdot\left(V_{1}^{n}-V_{2}^{n}\right)=0 .} \\
& \forall t \in[0,1], \quad f\left(t V_{1}^{n}+(1-t) V_{2}^{n}\right) \geqslant t f\left(V_{1}^{n}\right)+(1-t) f\left(V_{2}^{n}\right) .
\end{aligned}
$$

We write $\eta_{n}:=\left|V_{1}^{n}-V_{2}^{n}\right|\left(\lim \eta_{n}=0\right)$ and $W^{n}:=\frac{1}{\eta_{n}}\left(V_{1}^{n}-V_{2}^{n}\right)$; since this is a unit vector, we can assume that $W^{n}$ converges to some limit $W$ as $n \rightarrow \infty$.

Hence the function $j(t):=f\left(V_{1}^{n}+t \eta_{n} W^{n}\right)$ satisfies $j^{\prime}(0)=j^{\prime}(1)$. As a consequence, there exist $t_{n} \in(0,1)$ where $j^{\prime \prime}\left(t_{n}\right)=0$, by Rolle's Theorem. Since $j(t) \geqslant t j(0)+(1-$ $t) j(1)$ for all $t \in[0,1]$, we must have $j^{\prime \prime}(0) \geqslant 0, j^{\prime \prime}(1) \geqslant 0$, and $j^{\prime \prime}(t)$ must change sign twice in $[0,1]$; therefore, there exist $\tau_{n} \in(0,1)$ such that $j^{\prime \prime \prime}\left(\tau_{n}\right)=0$.

Returning to $f$, we have for all $n$ :

$$
\begin{aligned}
d^{2} f\left(V_{1}^{n}+t \eta_{n} W^{n}\right) W^{n} \cdot W^{n} & =0, \\
d^{3} f\left(V_{1}^{n}+\tau_{n} \eta_{n} W^{n}\right) W^{n} \cdot W^{n} \cdot W^{n} & =0 .
\end{aligned}
$$

In the limit, as $n \rightarrow \infty$, recall that $V_{1}^{n} \rightarrow V:=\nabla u(y), \eta_{n} \rightarrow 0$, so that

$$
d^{2} f(V) W \cdot W=0=d^{3} f(V) W \cdot W \cdot W
$$

with $|W|=1$. This contradicts Hypothesis $(\mathrm{H})$ on $f$.

Step 4 . By the previous step there is an open subset $\Omega_{2}$ such that $\partial u\left(x_{0}\right)$ has empty interior for all $x_{0} \in \Omega_{2}$, i.e. all subdifferentials are points or line segments. Therefore,

$$
\int_{B\left(x_{0}, \varepsilon\right)} \operatorname{det} d^{2} u d x=\int_{\partial u\left(B\left(x_{0}, \varepsilon\right)\right)} d p=\left|\partial u\left(B\left(x_{0}, \varepsilon\right)\right)\right| \rightarrow 0
$$

as $\varepsilon \rightarrow 0$ (see, e.g., [11, Corollary 24.5.1] for such convergence results). Consequently, the singular part of the Radon measure $d^{2} u$ is zero in $\Omega_{2}$; since $u$ is strictly convex, the regular part is not identically zero in $\Omega_{2}$ (in fact the support of the regular part is $\Omega_{2}$ ).

Therefore we can choose a point in $\Omega_{2}$ such that $d^{2} u$ is defined in the classical sense and non-zero, and we fix this point as the origin of coordinates. By subtracting an affine function and translating $f$ we may assume that $u(0)=0$ and $\nabla u(0)=0$.

We claim that there exist two one-dimensional strictly convex functions $\phi_{1}, \phi_{2}$, defined in a neighbourhood of 0 , such that $\phi_{i}(0)=\phi_{i}^{\prime}(0)=0$ and $u\left(x_{1}, x_{2}\right)-\phi_{1}\left(x_{1}\right)-$ $\phi_{2}\left(x_{2}\right)$ is a convex function.

Indeed, let $a>0$ be small enough in order to ensure that the square $[-a, a]^{2}$ is included in $\Omega_{1}$. For each given $x_{2} \in[-a, a], u\left(\cdot, x_{2}\right)$ is a convex function defined on $[-a, a]$; its second-order derivative is a positive measure $\partial_{11} u\left(\cdot, x_{2}\right)$. We define a measure $\mu$ on $[-a, a]$ by

$$
\forall(\alpha, \beta) \subset[-a, a], \quad \begin{aligned}
\mu(\alpha, \beta) & =\inf _{x_{2} \in[-a, a]} \partial_{11} u\left((\alpha, \beta), x_{2}\right) \\
& =\inf _{x_{2} \in[-a, a]}\left(\partial_{1} u\left(\beta, x_{2}\right)-\partial_{1}\left(\alpha, x_{2}\right)\right) .
\end{aligned}
$$

The latter infimum is necessarily positive, since a zero value would contradict the strict convexity of $u$; hence $\mu$ is a strictly positive measure. Let $A>0$ be a large number to 
be chosen in a while, and define $\phi_{1}:[-a, a] \rightarrow \mathbb{R}$ as the unique strictly convex function with second derivative $\mu / A$, and satisfying $\phi_{1}(0)=\phi_{1}^{\prime}(0+)=0$. Since $u$ is differentiable at $x=0, \phi_{1}$ is differentiable at 0 , and $\phi_{1}^{\prime}(0)=0$.

Since $\phi_{1}^{\prime \prime} \leqslant A \partial_{11} u\left(\cdot, x_{2}\right)$ for all $x_{2} \in[-a, a]$, we see that $u\left(x_{1}, x_{2}\right)-A \phi_{1}\left(x_{1}\right)$ is convex with respect to $x_{1}$, for $x_{2}$ fixed. Obviously, that does not mean that $u-A \phi_{1}$ is convex; but we can reduce to a smaller neighbourhood $[-b, b]^{2} \subset[-a, a]^{2}$ where $u-\phi_{1}$ is strictly convex. Indeed, let us note

$$
\gamma(x, y)=\gamma\left(x_{1}, x_{2}, y_{1}, y_{2}\right):=u(x)+u(y)-2 u((x+y) / 2) .
$$

Since $u$ is stricly convex, $\gamma(x, y)>0$ if $x \neq y$. We assume now that $A$ satisfies

$$
A>\frac{\partial_{11} u(0) \partial_{22} u(0)}{\operatorname{det} d^{2} u(0)}
$$

(note that it is possible to find such a number, by the assumption that $\operatorname{det} d^{2} u(0)$ is nonzero), and we claim that there exists $b \in(0, a)$ such that

$$
\forall x, y \in[-b, b]^{2} \text {, with } x \neq y \quad \gamma\left(x_{1}, x_{2}, y_{1}, x_{2}\right)<A \gamma(x, y) .
$$

Indeed, if not, we can find sequences $\left(x^{n}\right),\left(y^{n}\right), x^{n} \neq y^{n}$ for all $n$, both converging to 0 , such that $\gamma\left(x_{1}^{n}, x_{2}^{n}, y_{1}^{n}, x_{2}^{n}\right) \geqslant A \gamma\left(x^{n}, y^{n}\right)$. A simple Taylor expansion near 0 gives $\partial_{11} u(0)\left(x_{1}^{n}-y_{1}^{n}\right)^{2} \geqslant A d^{2} u(0) \cdot\left(x^{n}-y^{n}\right) \cdot\left(x^{n}-y^{n}\right)+\mathrm{o}\left(\left|x^{n}\right|+\left|y^{n}\right|\right)$, or

$$
\left(1-\frac{1}{A}\right) \partial_{11} u(0) X_{1}^{2}+2 \partial_{12} u(0) X_{1} X_{2}+\partial_{22} u(0) X_{2}^{2} \leqslant 0
$$

with $X_{i}=\lim _{n \rightarrow \infty}\left(x_{i}^{n}-y_{i}^{n}\right) /\left|x^{n}-y^{n}\right|$. Therefore, the discriminant of this quadratic form must be non-positive, i.e. $\partial_{12} u(0)^{2} \geqslant\left(1-\frac{1}{A}\right) \partial_{11} u(0) \partial_{22} u(0)$. But this contradicts (29), as a little computation shows. This ends the proof of (30).

Since $u-A \phi_{1}$ is convex with respect to $x_{1}$, we know that for each $x, y(x \neq y)$,

$$
\begin{aligned}
\phi\left(x_{1}\right)+\phi\left(y_{1}\right)-2 \phi\left(\frac{x_{1}+y_{1}}{2}\right) & \leqslant \frac{1}{A}\left[u\left(x_{1}, x_{2}\right)+u\left(y_{1}, x_{2}\right)-2 u\left(\frac{x_{1}+y_{1}}{2}, x_{2}\right)\right] \\
& \leqslant \frac{1}{A} \gamma\left(x_{1}, y_{1}, x_{2}, x_{2}\right) \\
& <\gamma(x, y)=u(x)+u(y)-2 u((x+y) / 2) .
\end{aligned}
$$

Hence $u-\phi_{1}$ is strictly convex.

To obtain $\phi_{2}$, we merely repeat the same process, starting from the strictly convex function $u-\phi_{1}$ and using the second coordinate.

Step 5 . We now obtain a contradiction with the minimality of $u$. Indeed, let us choose three positive real numbers $\alpha_{1}, \alpha_{2}, \beta$. If $\beta$ is small enough, the set

$$
\omega_{\beta}:=\left\{x=\left(x_{1}, x_{2}\right) ; \alpha_{1} \phi_{1}\left(x_{1}\right)+\alpha_{2} \phi_{2}\left(x_{2}\right)<\beta\right\}
$$

is a neighbourhood of 0 included in the domain where $u-\phi_{1}\left(x_{1}\right)-\phi_{2}\left(x_{2}\right)$ is convex. Note that for $\varepsilon>0$ small enough, the function $u-\varepsilon\left(\alpha_{1} \phi_{1}\left(x_{1}\right)+\alpha_{2} \phi_{2}\left(x_{2}\right)\right)$ is also convex. 
Hence, the function

$$
v_{\varepsilon}:=\max \left(u, u-\varepsilon\left(\alpha_{1} \phi_{1}\left(x_{1}\right)+\alpha_{2} \phi_{2}\left(x_{2}\right)-\beta\right)\right)
$$

is convex, defined on $\Omega$, and differs from $u$ only in $\omega_{\beta}$. We must therefore have $F\left(v_{\varepsilon}\right) \geqslant F(u)$, which is

$$
\int_{\omega_{\beta}} f\left(\nabla v_{\varepsilon}\right)-f(\nabla u) \geqslant 0 .
$$

By choosing $\varepsilon$ small enough, we get from a Taylor expansion

$$
\int_{\omega_{\beta}} d^{2} f(\nabla u) \nabla\left(v_{\varepsilon}-u\right) \cdot \nabla\left(v_{\varepsilon}-u\right) \geqslant 0
$$

(the first order term vanishes by (27)). This can also be written

$$
\int_{\omega_{\beta}} d^{2} f(\nabla u) \nabla \psi \cdot \nabla \psi \geqslant 0,
$$

where $\psi(x):=\alpha_{1} \phi_{1}\left(x_{1}\right)+\alpha_{2} \phi_{2}\left(x_{2}\right)$. Recall that this inequality holds for all numbers $\alpha_{i}>0(i=1,2)$, if $\beta$ is small enough, and for any coordinate system $\left(x_{1}, x_{2}\right)$. Hence, we can assume that $d^{2} f(0)$ is just a diagonal matrix $\left(\begin{array}{cc}\lambda_{1} & 0 \\ 0 & \lambda_{2}\end{array}\right)$, with for instance $\lambda_{1}<0$ (recall that $f$ is nowhere convex). Since the map $x \mapsto d^{2} f(\nabla u(x))$ is continuous and $\left|\omega_{\beta}\right| \rightarrow 0$ as $\beta \rightarrow 0$, we have $\lim _{\beta \rightarrow 0} c(\beta)=0$, if we write $c(\beta)=\sup _{\omega_{\beta}} \mid d^{2} f(\nabla u(x))-$ $d^{2} f(0) \mid$. Hence, we get from (31):

$$
\lambda_{1} k_{1}(\alpha, \beta)+\lambda_{2} k_{2}(\alpha, \beta) \geqslant-c(\beta)\left(k_{1}+k_{2}\right)(\alpha, \beta),
$$

where $\alpha=\left(\alpha_{1}, \alpha_{2}\right)$ and $k_{i}(\alpha, \beta):=\alpha_{i}^{2} \int_{\omega_{\beta}} \phi_{i}^{\prime}\left(x_{i}\right)^{2} d x_{1} d x_{2}$.

Recall that $\phi_{i}$ is a strictly convex function minimal at 0 . Hence, the function $s \in$ $\mathbb{R} \mapsto \phi_{i}(s)$ sign $s$ is increasing and has a reciprocal function that we denote $\eta_{i}$; note that $\eta_{i}(0)=0$, but the derivative $\eta_{i}^{\prime}(s)=\operatorname{sign} s / \phi_{i}^{\prime} \circ \eta_{i}(s)$ is infinite at $s=0$. Hence, if $m>0$ is any number, we have (dropping indices $i$ for short)

$$
\begin{aligned}
\alpha^{2} \int_{\alpha \phi(x)<m} \phi^{\prime}(x)^{2} d x & \left.=\alpha \int_{-m}^{m} \phi^{\prime} \circ \eta\left(\frac{t}{\alpha}\right) \operatorname{sign} t d t \quad \text { [set } t=\alpha \phi(x) \operatorname{sign} x\right] \\
& =\int_{-m}^{m} \frac{|t|}{\left|\frac{t}{\alpha} \eta^{\prime}\left(\frac{t}{\alpha}\right)\right|} d t \\
& >\int_{-m}^{m} \frac{|t|}{\left|\eta\left(\frac{t}{\alpha}\right)\right|} d t \rightarrow+\infty \quad \text { as } \alpha \rightarrow \infty .
\end{aligned}
$$

(The last inequality is a consequence of $t \eta^{\prime}(t)<\eta(t)$, equivalent to $x \phi^{\prime}(x)>\phi(x)$, which follows from the convexity of $\phi$.) 
Hence, $k_{1}(\alpha, \beta)$ tends to $\infty$ as $\alpha_{1}$ tends to infinity. As a consequence, it is possible to find sequences $\left(\alpha_{n}\right) \subset(0, \infty)^{2},\left(\beta_{n}\right) \subset(0, \infty)$ such that $\beta_{n}$ and $\left(k_{2} / k_{1}\right)\left(\alpha_{n}, \beta_{n}\right)$ converge to 0 . Replacing these numbers in (32), and letting $n$ going to infinity, we get $\lambda_{1} \geqslant 0$, a contradiction.

This concludes the proof of Lemma 5.

\section{Acknowledgement}

The authors wish to express their gratitude to Professors G. Buttazzo, B. Kawohl, and $\mathrm{H}$. Berestycki for bringing this problem to their attention, and A. Burchard and A. Colesanti, as well as those mentioned above, for several interesting discussions.

The research of the second author was supported by the EC Human Capital and Mobility Project ERBCHRXCT940494.

\section{REFERENCES}

[1] Brock F., Ferone V., Kawohl B., A symmetry problem in the calculus of variations, Calc. Var. Partial Differential Equations 4 (1996) 593-599.

[2] Buttazzo G., Ferone V., Kawohl B., Minimum problems over sets of concave functions and related questions, Math. Nachrichten 173 (1993) 71-89.

[3] Evans L.C., Gariepy R.F., Measure Theory and Fine Properties of Functions, Studies in Applied Mathematics, CRC Press, 1992.

[4] Goldstine H.H., A History of the Calculus of Variations from the 17th through the 19th Century, Springer-Verlag, Heidelberg, 1980.

[5] Guasoni P., Problemi di ottimizzazione di forma su classi di insiemi convessi, Tesi di Laurea, Pisa, 1996.

[6] Benoist J., Hiriart-Urruty J.B., What is the subdifferential of the closed convex hull of a function?, SIAM J. Math. Anal. 27 (6) (1996) 1661-1679.

[7] Lachand-Robert T., Peletier M.A., Extremal points of a functional on the set of convex functions, Proc. Amer. Math. Soc. 127 (1999) 1723-1727.

[8] Lachand-Robert T., Peletier M.A., Newton's problem of the body of minimal resistance in the class of convex developable functions, in preparation.

[9] Newton I., Philosophiae Naturalis Principia Mathematica, 1686.

[10] Reed M., Simon B., Methods of Mathematical Physics, Vol. I, Academic Press, 1980.

[11] Rockafellar R.T., Convex Analysis, Princeton University Press, 1970.

[12] Schneider R., Convex Bodies: The Brunn-Minkowski Theory, Cambridge University Press, 1993.

[13] Zamfirescu T., Baire category in convexity, Atti Sem. Mat. Fis. Univ. Modena 39 (1991) $139-164$.

[14] Zamfirescu T., Nearly all convex bodies are smooth and strictly convex, Monatsh. Math. 103 (1987) 57-62. 